\title{
Puritanisme sexuel et capitalisme numérique
}

entretien avec Susanna Paasonen, Kylie Jarrett et Ben Light

réalisé et traduit par Florian Vörös (Université de Lille, Gériico)

Revue française de socio-économie, ${ }^{\circ}$ 25, 2020, p. 167-174.

Trois chercheur-es spécialistes des médias numériques signent ensemble aux presses du Massachussets Institute of Technology un ouvrage consacré à la régulation de la sexualité sur les réseaux socio-numériques : NSFW. Sex, Humor, and Risk in Social Media [Paasonen, Jarrett, Light, 2019]. Susanna Paasonen, professeure à l’Université de Turku (Finlande), est connue pour son travail sur les pornographies numériques, dont elle explore les dimensions affectives dans une perspective queer [Paasonen, 2011, 2014]. Kylie Jarrett, professeure associée à l’Université de Maynooth (Irlande), s’intéresse, dans une perspective féministe marxiste, à l'exploitation du travail féminin par les plateformes de sociabilité en ligne [Jarrett, 2016, 2017]. Ben Light, professeur à l’Université de Salford (Angleterre) étudie quant à lui les sociabilités en ligne depuis la sociologie des techniques [Light, 2014]. Cet entretien revient sur les enjeux socio-économiques de la circulation des contenus sexuels en ligne.

Not Safe For Work (NSFW) est une expression native des réseaux socio-numériques qui désigne avec humour les images à ne pas regarder devant ses collègues. Dans votre livre [Paasonen, Jarrett, Light, 2019], vous insistez sur le fait que marquer un contenu comme « NSFW » opère à la fois comme un avertissement et comme une invitation à cliquer. Vous décrivez aussi comment, d'expression vernaculaire, NSFW est progressivement devenue une catégorie de régulation pour des entreprises telles que Google, Facebook ou Tumblr. Pouvez-vous nous présenter la démarche de ce livre et le contexte dans lequel il s'inscrit?

Notre livre est à la fois une enquête sur les usages ordinaires de la catégorie NSFW et une critique de l'amalgame entre sexualité et danger dans les politiques de régulation des réseaux socio-numériques. Notre point de départ a été l'extraction et l'analyse de grands corpus de tweets contenant l'acronyme NFSW et le mot-dièse \#NSFW. Mais ce livre s'intéresse plus largement à la régulation de la sexualité au sein de l'économie numérique, à travers des études de cas sur l'humour et la sexualité au travail, le harcèlement sexiste ou encore les dick pics (photos de pénis - sollicitées ou non).

Les controverses relatives à la régulation de la sexualité en ligne se sont intensifiées alors que nous étions en train d'écrire le livre. En décembre 2018, la plateforme de microblogging Tumblr, longtemps connue pour sa clémence envers la nudité, la sexualité et la pornographie, ainsi que comme un espace de sociabilité important pour les minorités de genre et de sexualité, a durci ses règles et s'est mise à bannir les contenus dits « NSFW ». L'entreprise Facebook, déjà connue pour son conservatisme, a également durci ses règles, en excluant non seulement les images de nudité, mais aussi les expressions textuelles de désir sexuel. Le code de bonne conduite («community standards») édicté par Facebook exclut en effet tout 
contenu qui «facilite, encourage ou coordonne la rencontre sexuelle entre adultes ». Dans ce contexte, les publications sexuelles, et les expériences partagées auxquelles elles ouvrent, sont de plus en plus repoussées en dehors des réseaux socio-numériques.

La sexualité a été au cour du développement du Minitel dans les années 1980 puis du web dans les années 1990. Dans votre livre vous rappelez que les systèmes de paiement sécurisé par carte de crédit, la vidéo en streaming, les bannières de publicité, les popups et les technologies de suivi de la souris ont d'abord été développés pour, et utilisés par des sites pornographiques. Que sait-on de l'économie de la pornographie en ligne aujourd'hui ? Quelle place occupe-t-elle dans l'économie du web ?

Cela fait un certain temps que l'industrie du porno n'est plus à la pointe de l'innovation technique et économique. Les sites d'agrégation de vidéos pornographiques tels que PornHub imitent dans les grandes lignes les principes de fonctionnement de YouTube. Pendant ce temps-là, les géants du numérique tels que Facebook, Google/Alphabet et Apple, par lesquels passe une grande partie du trafic de données issu des réseaux socio-numériques, s'efforcent de filtrer et de supprimer les contenus pornographiques et, par amalgame, un large éventail de contenus considérés comme sexuels. Les outils de cette gouvernance par obstruction sont les contrats de licence utilisateur final, par lesquels les entreprises qui commercialisent des applications délimitent le cadre de leur utilisation légale et, en amont, les directives des grands catalogues d'applications mobiles, tels que Google Play et Apple Store, qui demandent aux applications de ne pas autoriser la nudité, la sexualité et toute une série d'autres contenus jugés offensants. Ces restrictions ont pour effet d'isoler les sites pornographiques du reste de l'économie du numérique. Cette dichotomie entre réseaux socionumériques et pornographie laisse aussi de moins en moins d'espace pour la circulation en ligne des contenus sexuels non-pornographiques : outils d'éducation sexuelle, informations concernant les minorités sexuelles, documentaires sur les cultures sexuelles, art érotique, etc. [Tiidenberg, van der Nagel, 2020].

La vidéo pornographique en ligne continue bien sûr de générer des revenus, mais le partage de ces derniers est de plus en plus inégalitaire. Le groupe MindGeek, basé à Montréal, possède aujourd'hui les sites PornHub, YouPorn, RedTube et Tube8, ce qui lui confère un quasi monopole sur la diffusion de pornographie audiovisuelle en ligne. Ce groupe a aussi racheté plusieurs grands studios qui s'étaient retrouvés affaiblis par l'abondance de pornographie mise à disposition " gratuitement » en ligne, la plupart du temps illégalement. Autrement dit, MindGeek pirate désormais le contenu des studios qu'il possède, tout en incitant les utilisateurs à payer pour accéder à ces contenus par le biais d'une adhésion « premium ». Sa source principale de revenus reste cependant, selon toute vraisemblance, le recueil de données personnelles et la vente d'espaces publicitaires ciblés. Ces revenus sont très peu partagés avec les travailleurs-ses du sexe qui produisent les contenus.

À l'instar des autres industries créatives, le secteur de la pornographie se transforme en une économie à la tâche ou gig economy [Berg, 2016]. Les réalisateurs-trices, producteurs-trices et acteurs-trices sont désormais considéré-e-s par les studios comme des «talents » 
prestataires de services, ce qui a des conséquences sur leurs conditions de travail et d'emploi. Prenons l'exemple de la rémunération de ces différentes prestataires : du fait de l’opacité des modalités de rémunération, il est difficile d'évaluer ce qui constitue aujourd'hui une rémunération juste pour le tournage d'une scène. Ce qui est certain, c'est que la mise en concurrence des «talents » maintient ces rémunérations à un faible niveau. Pour joindre les deux bouts, de nombreux-euses acteurs-trices pornographiques se mettent donc à proposer des services d'escorte, ce qui n'était pas la règle auparavant. De leur côté, les escortes peuvent tourner des scènes pornos pour renforcer leur image de marque et ainsi augmenter leurs tarifs. Les contrats d'exclusivité à long terme avec les studios ont pratiquement disparu et les artistes se voient confier la tâche de construire leur statut de star de manière indépendante. Ce travail d'autopromotion en ligne se fait notamment via Twitter, une des rares plateformes de sociabilités grand public à n'avoir pas encore interdit les contenus pour adultes.

\section{Cette réglementation accrue de l'expression en ligne a-t-elle une explication économique?}

La logique économique qui sous-tend ces restrictions est peu évidente. On sait que certaines marques qui souhaitent éviter que la publicité pour leurs produits jouxte des contenus pornographiques peuvent exercer des pressions [Lambiase, 2003/2012 ; McStay, 2016]. Mais les contenus sexuels ne font pas que repousser, ils attirent aussi. Ces contenus jouent un rôle important dans la stimulation des flux d'attention qui nourrissent le marché de la publicité en ligne. Il paraît illogique sur le plan économique de supprimer ou de limiter la visibilité d'un contenu pourtant capable de générer les vues et les clics sur lesquels s'appuie aujourd'hui l'économie de la donnée. Le cas de Tumblr illustre bien cette absurdité économique : début 2019, trois mois après l'interdiction des contenus sexuels sur la plateforme, le trafic d'utilisateurs avait déjà chuté de près de $30 \%$. Cette baisse a eu des effets sur le cours de l'action du site en bourse, qui n'a cessé de diminuer. Tumblr avait d'abord été racheté par Yahoo en 2013 pour 1,1 milliard de dollars, puis par Verizon dans le cadre du rachat du groupe Yahoo en 2017. En 2019, Tumblr a été racheté par Automattic (propriétaire de Wordpress) pour moins de 3 millions de dollars US. Il y a clairement un lien de cause à effet entre l'interdiction des contenus pornographiques, la diminution du trafic et le déclin économique de Tumblr - même si cette plateforme n’a jamais été très rentable, l'activité en ligne y étant difficile à monétiser.

Ces restrictions suivent en fait plutôt une logique politique. En interdisant les contenus sexuels explicites, Tumblr pérennise la présence de son application sur les catalogues App Store et Google Play, en s'alignant sur leurs politiques de contenus. Elle se conforme aussi aux évolutions législatives relatives à la diffusion de vidéos pédopornographiques et issues du trafic d'êtres humains. Les lois états-uniennes Stop Enabling Sex Traffickers Act (SESTA) et Allow States and Victims to Fight Online Sex Trafficking Act (FOSTA) adoptées en avril 2018 imposent de sérieuses limitations juridiques aux plateformes quant à la publicité du commerce sexuel en ligne. Celles qui y contreviennent s'exposent désormais à leur exclusion des catalogues d'applications en ligne, au boycott par les annonceurs et à de possibles 
poursuites judiciaires. Ces dispositions visent en principe à protéger les personnes vulnérables contre les abus de "l'industrie du sexe». Dans la pratique, tout contenu à caractère sexuel devient potentiellement assimilable à de la violence ${ }^{1}$. Cet amalgame a des effets délétères sur l'activité économique des travailleurs-ses du sexe, ainsi que sur les personnes qui prennent part à des cultures sexuelles marginalisées, pour qui la sociabilité sur des sites comme Tumblr avait une valeur inestimable. Certaines plateformes, comme Twitter, parviennent toutefois à échapper à cette logique, probablement en raison de leur antériorité et de leur poids économique.

\section{Comment penser la relation entre les valeurs, au sens culturel et moral du terme, et la création de valeur économique en ligne ? Comment les cultural studies, la théorie queer et les théories des affects peuvent-elles aider à penser cette relation ?}

Ce durcissement des politiques de régulation est un phénomène indissociablement économique et culturel. Pour le comprendre, il faut commencer par rappeler que différents systèmes de valeurs culturelles peuvent coexister avec différents modèles de création de valeur économique. Cette coexistence devient conflictuelle lorsque ce qui va de soi pour certain-e-s apparaît étrange à d'autres. Dans un contexte où les réseaux socio-numériques et, plus généralement l'économie du web, restent fortement centrés sur les États-Unis, les valeurs culturelles spécifiques à ce contexte deviennent, via les codes de bonne conduite ( « community standards ») édictés par ces entreprises, des normes mondiales qui s'appliquent à la planète entière. Les États-Unis ont beau être le terreau de cultures sexuelles diverses et radicales, rien de tout cela ne transparaît vraiment dans les politiques de régulation de contenu des réseaux socio-numériques.

Le puritanisme sexuel de Facebook et consorts peut être envisagé comme une «structure de sentiment résiduelle » au sens de Raymond Williams [Williams, 1977] : c'est un système de valeurs culturelles qui appartient au passé mais qui ne cesse de résonner dans le présent. Selon ce système de valeurs, la sexualité est risquée, la nudité est dangereuse et il vaudrait mieux les garder hors de notre vue. Aussi ridicules qu'elles puissent nous sembler, ces normes irriguent autant les règles des réseaux socio-numériques que les directives des catalogues d'application mobiles. Tout cela est lié aux intérêts des annonceurs car la plupart des grandes marques - telles que Nike, H\&M, Starbucks ou McDonalds - ne souhaitent pas que leurs publicités apparaissent à côté d'images pornographiques. Le principe économique prime sur tout le reste, mais ce principe se formule dans les termes d'un sens commun, d'un «bon sens », qui voudrait que l'objectif de sécurité justifie évidemment le filtrage de tout contenu offensant.

La théorie queer [Rubin, 1984 ; Berlant et Warner, 1998] aide à comprendre l'émergence et le déploiement des normes sexuelles, ainsi que les résistances dont celles-ci font l'objet. Les théories des affects [Hillis, Paasonen, Petit, 2015 ; Sampson, Maddison, Ellis, 2018] aident

$1 \mathrm{NdT}$ : On retrouve cet amalgame entre sexualité explicite et violence dans le droit français. Au nom de la lutte contre les violences conjugales, la loi du 30 juillet 2020 impose aux sites pornographiques de mettre en place un contrôle de l'âge de leurs clients. 
quant à elles à cartographier les intensités en jeu dans la circulation et l'accumulation des données, dans la captation de l'attention des publics, ainsi que dans la formation des attachements et des tensions en ligne. Dans le cas des contenus NSFW, les intensités affectives se manifestent par des combinaisons ambivalentes de dégoût, d'amusement, d'intérêt, d'excitation, de choc, de titillation, de haine et de plaisir qui, dans leur ambiguïté et leur complexité, ne peuvent être réduites à une opposition binaire entre sentiments négatifs et positifs - pas plus que les sexualités en ligne ne peuvent d'ailleurs être simplement placées d’un côté ou de l'autre de la barrière qui sépare la sécurité du risque, malgré tous les efforts que l’on peut déployer pour tracer et consolider cette frontière.

La marginalisation de toute expression sexuelle sur les réseaux socio-numériques témoigne d'une profonde dévalorisation de la sexualité. La sexualité est non seulement considérée comme risquée et offensante mais, plus fondamentalement, comme sans valeur. C'est ainsi que la sexualité est de plus en plus exclue des réseaux socio-numériques. Nous pensons qu’il s’agit là d'une tendance à laquelle il faut résister.

\section{Comment les plateformes s'y prennent-elles pour éviter que les contenus catégorisés comme « NSFW " apparaissent sur nos écrans ? Sur quel travail s'appuie l'activité de modération?}

La modération des publications sur les réseaux socio-numériques se fait d’abord par filtrage algorithmique automatisé, à partir de logiciels de reconnaissance de mots et d'images. Ces derniers ont tendance à cibler les corps féminins davantage que les corps masculins. Leurs algorithmes ont été développés pour reconnaître les images de corps féminins - et, plus spécifiquement, de corps féminins blancs. Les seins sont ainsi plus facilement détectés en tant que « contenus sexuels offensants » que les pénis. Cela nous renvoie au problème plus large du biais hétérosexuel masculin blanc dans la conception des logiciels informatiques [Gehl et al., 2017].

La modération dépend aussi des signalements des usager-ère-s et des travailleurs-euses de l'industrie de la modération de contenu en ligne [Gillespie, 2018]. Leur travail, souvent délocalisé dans des pays où la main d'œuvre est bon marché, consiste à passer en revue les publications signalées et à prendre des décisions à leur sujet. Sarah T. Roberts documente ce « sale boulot » de la culture numérique dans l'enquête qu'elle a menée au sein de l'industrie de la modération aux Philippines [Roberts, 2019]. Il ne s’agit pas seulement d'évincer des tétons et des photos de pénis, mais aussi des vidéos de décapitation ou de cruauté envers les animaux. C’est donc un travail particulièrement éprouvant.

La catégorie NSFW est quasi exclusivement appliquée aux contenus sexuels - qui sont ainsi marqués comme dangereux - plutôt qu'aux contenus de type violent ou haineux par exemple. Cet usage de la catégorie NSFW participe d'une définition de la nudité, du sexe et de la sexualité comme des réalités problématiques à exclure des réseaux socio-numériques. 


\section{Les politiques de contenu des réseaux socio-numériques tendent à assimiler la pornographie à la haine et au harcèlement en ligne. En quoi le féminisme marxiste permet une analyse socio-économique de ces phénomènes ?}

En travaillant à partir du féminisme marxiste, nous considérons dans ce livre la haine et le harcèlement en ligne comme des pratiques encastrées dans des contextes socio-économiques plus larges. Pour lutter contre le sexisme et la misogynie en ligne, les outils techniques de la modération de contenus - tels que les drapeaux, balises et formulaires de signalement - ne suffisent pas; il faut une transformation socio-économique plus profonde. Ce qui rend le travail des femmes dangereux dans les environnements numériques, ce n'est pas le visionnage de contenus sexuels explicites, mais la culture capitaliste et masculiniste qui y prédomine.

Pour comprendre le masculinisme en ligne, il faut se rappeler que le capitalisme numérique sollicite des qualités - telles que la communication et la flexibilité - qui ont historiquement été construites comme féminines et assimilées à un "travail de femme » [Adkins, 2001 ; Jarrett, 2016, 2017 ; Morini, 2007]. L’essor du capitalisme numérique est contemporain de la féminisation du travail rémunéré, du déclin de l'emploi stable et du détricotage des filets de sécurité sociale. Ces transformations posent un défi particulier au modèle de masculinité hégémonique fondé sur le travail. Les compétences valorisées dans le capitalisme numérique sont aussi par ailleurs celles de la «masculinité geek » [Kendall 2011 ; Massanari 2015], basée sur une culture de la confrontation en ligne (le trolling), qui se développe notamment via les plateformes reddit et 4 chan $^{2}$. Dans ce contexte, nous considérons la haine misogyne et le harcèlement sexiste en ligne comme des pratiques de défense active des privilèges masculins au sein du capitalisme numérique, contre la concurrence des femmes [Ging et Siapera, 2019 ; Jane 2016].

Les espaces numériques se sont construits autour des idéaux libéraux, voire libertariens, de la libre communication et de la méritocratie, selon lesquels les privilèges seraient le fruit du travail, plutôt que des déterminismes et des hiérarchies sociales [Reagle, 2013]. Selon cette perspective, nous serions libres de participer ou non à des environnements numériques et notre succès ne dépendrait que de nos talents techniques et linguistiques. Le fait que l'accès aux codes de la sociabilité geek puisse être limité par le genre, la race ou la classe, et que les normes soient préétablies en fonction de ces rapports sociaux, n’entre pas en ligne de compte dans ce raisonnement. Le non-respect des normes est alors envisagé comme un échec individuel, ce qui marque la personne qui échoue comme déviante et ouvre la voie à l'abus, dans une logique de reproduction du système.

Ce sont en fait le masculinisme et le capitalisme qui rendent périlleux le travail des femmes dans les environnements numériques. Et ce sont précisément ces facteurs structurels que l'on oublie lorsque l'on fait de la sexualité explicite la cause du sexisme en ligne. C'est aussi pour

2 NdT: Réseaux socio-numériques états-uniens initialement consacrés à la programmation informatique (Reddit) et à la culture manga (4chan), régulièrement utilisés pour la coordination d’actions sexistes, racistes, transphobes et grossophobes. 
cela qu'il est urgent de repenser la régulation du web au-delà de l'amalgame entre sexualité et danger.

\section{Bibliographie}

AdKins L. (2001), "Cultural feminization: 'Money, sex and power' for women », Signs: Journal of Women in Culture and Society, vol. 26, n 3, p. 669-695.

BERLANT L., WARner M. (2018[1998]), "Sexe en public », trad. de Maxime Cervulle et Clémence Garrot, Questions de communication, n 33, p. 111-133.

BERG H. (2016), “'A scene is just a marketing tool': alternative income streams in porn's gig economy », Porn Studies, vol. 3, n 2, p. 160-174.

GeHL R. W., Moyer-Horner L., Yeo S. K. (2017), « Training computers to see internet pornography. Gender and sexual discrimination in computer vision science », Television \& New Media, vol. 18, n 6, p. 529-547.

GILLESPIE T. (2018), Custodians of the Internet, New Haven, Yale University Press.

GING D., SIAPERA E. (2019), Gender hate online: Understanding the new anti-feminism. Basingstoke, Palgrave.

JANE E. (2016), Misogyny online: A short (and brutish) history, Londres, SAGE.

JARretT K. (2016), The Digital Housewife. Feminism, Labor and Digital Media, New York, Routledge.

JARRETT K. (2017), « Le travail immatériel dans l'usine sociale : une critique féministe », trad. d'Hélö̈se Noisette et Florian Vörös, Poli - Politique de l'image, n 13, p. 12-25.

Kendall L. (2011), "'White and Nerdy': Computers, Race and the Nerd Stereotype », Journal of Popular Culture, n 3, p. 505-524.

Hillis K., PaAsonen S., Petit M. (dir.) (2015), Networked Affect, Cambridge, Mass., MIT Press.

LAMBiAse J. J. (2003/2012), « Sex-Online and in Internet Advertising », in T. Reichert and J.J. LAmbiase (dir), Sex in advertising: Perspectives on the erotic appeal, New York, Routledge, p. 247-272.

LigHT B. (2014), Disconnecting with Social Networking Sites, Basingstoke, Palgrave Macmillan.

McStay A. (2016), Digital Advertising, $2^{\mathrm{e}}$ édition, Londres, Palgrave Macmillan.

MASSANARI A. (2015), "\#Gamergate and The Fappening: How Reddit's algorithm, governance, and culture support toxic technocultures », New Media \& Society, vol. 19, n 3, p. 329-346.

MORINI C. (2007), « The feminization of labour in cognitive capitalism », Feminist review, ${ }^{\circ}$ 87, p. 40-59.

PAasonen S. (2011), Carnal Resonance. Affect and Online Pornography, Cambridge, Mass., MIT Press.

PAASONEN S., VÖRÖS F. (2014), "Affects et pornographies numériques. Entretien avec Susanna Paasonen », Poli - Politique de l’image n 9, 2014, p. 80-88.

PAASONEN S., JARRETT K., LIGHT B. (2019), NSFW. Sex, humor and risk in social media, Cambridge, Mass., MIT Press. 
REAGLE J. (2013), “'Free as in sexist?' Free culture and the gender gap », First Monday, http://firstmonday.org/ojs/index.php/fm/article/view/4291.

RoBerts S. T. (2020[2019]), Derrière les écrans. Les nettoyeurs du web à l'ombre des réseaux sociaux, trad. de Sophie Renaut, préface d'Antonio Casilli, Paris, La Découverte.

Rubin G. (2011[1984]), " Penser le sexe. Pour une théorie radicale de la politique de la sexualité ", trad. de Flora Bolter, Surveiller et jour. Anthropologie politique du sexe, Paris, Epel, p. 135-210.

SAMPSON T., MAdDison S., Ellis D. (2018), Affect and Social Media, Lanham, MD, Rowman \& Littlefield.

Timenberg K., Van Der Nagel E. (2020), Sex and Social Media, Bingley, Emerald Publishing.

WILLIAMS R. (1977), Marxism and Literature, Oxford, Oxford University Press. 